\title{
BMJ
}

\section{Clinical risk prediction for pre-eclampsia in nulliparous women: development of model in international prospective cohort}

\author{
Robyn A North, professor of maternal and fetal medicine, ${ }^{1}$ Lesley M E McCowan, professor of obstetrics and \\ gynaecology, ${ }^{2}$ Gustaaf A Dekker, professor of obstetrics and gynaecology, ${ }^{3}$ Lucilla Poston, professor of \\ maternal and fetal health, ${ }^{1}$ Eliza $\mathrm{H}$ Y Chan, research fellow, ${ }^{2}$ Alistair W Stewart, senior research fellow, ${ }^{4}$ \\ Michael A Black, senior lecturer, ${ }^{5}$ Rennae S Taylor, project manager, ${ }^{2}$ James I Walker, professor of obstetrics \\ and gynaecology, ${ }^{6}$ Philip N Baker, professor of obstetrics and gynaecology, ${ }^{7}$ visiting professor of obstetrics \\ and gynaecology, ${ }^{8}$ Louise C Kenny, professor of obstetrics ${ }^{9}$
}

'Division of Women's Health, King's
College London, London, United Kingdom

${ }^{2}$ Department of Obstetrics and Gynaecology, Faculty of Medical and Health Sciences, University of Auckland, Auckland, New Zealand

${ }^{3}$ Department of Obstetrics and Gynaecology, Lyell McEwin

Hospital, University of Adelaide, Adelaide, Australia

${ }^{4}$ Department of Epidemiology and Biostatistics, Faculty of Medical and Health Sciences, School of

Population Health, University of Auckland, Auckland

${ }^{5}$ Department of Biochemistry, University of Otago, Dunedin, New Zealand

${ }^{6}$ Leeds Institute of Molecular Medicine, University of Leeds, Leeds

${ }^{7}$ Faculty of Medicine and Dentistry, University of Alberta, Edmonton, Canada

${ }^{8}$ Department of Obstetrics and Gynaecology, University of Manchester, Manchester

${ }^{9}$ Anu Research Centre, Department of Obstetrics and Gynaecology, University College Cork, Republic of Ireland

Correspondence to: R A North robyn.north@kcl.ac.uk

Cite this as: $B M J$ 2011;342:d1875 doi:10.1136/bmj.d1875

\section{ABSTRACT}

Objectives To develop a predictive model for preeclampsia based on clinical risk factors for nulliparous women and to identify a subgroup at increased risk, in whom specialist referral might be indicated.

Design Prospective multicentre cohort.

Setting Five centres in Auckland, New Zealand; Adelaide, Australia; Manchester and London, United Kingdom; and Cork, Republic of Ireland.

Participants 3572 "healthy" nulliparous women with a singleton pregnancy from a large international study; data on pregnancy outcome were available for 3529 (99\%).

Main outcome measure Pre-eclampsia defined as $\geq 140 \mathrm{~mm} \mathrm{Hg}$ or diastolic blood pressure $\geq 90 \mathrm{~mm} \mathrm{Hg}$, or both, on at least two occasions four hours apart after 20 weeks' gestation but before the onset of labour, or postpartum, with either proteinuria or any multisystem complication. Preterm pre-eclampsia was defined as women with pre-eclampsia delivered before $37^{+0}$ weeks' gestation. In the stepwise logistic regression the comparison group was women without pre-eclampsia. Results Of the 3529 women, 186 (5.3\%) developed preeclampsia, including 47 (1.3\%) with preterm preeclampsia. Clinical risk factors at 14-16 weeks' gestation were age, mean arterial blood pressure, body mass index (BMI), family history of pre-eclampsia, family history of coronary heart disease, maternal birth weight, and vaginal bleeding for at least five days. Factors associated with reduced risk were a previous single miscarriage with the same partner, taking at least 12 months to conceive, high intake of fruit, cigarette smoking, and alcohol use in the first trimester. The area under the receiver operating characteristics curve (AUC), under internal validation, was 0.71 . Addition of uterine artery Doppler indices did not improve performance (internal validation AUC 0.71). A framework for specialist referral was developed based on a probability of pre-eclampsia generated by the model of at least $15 \%$ or an abnormal uterine artery Doppler waveform in a subset of women with single risk factors.
Nine per cent of nulliparous women would be referred for a specialist opinion, of whom $21 \%$ would develop preeclampsia. The relative risk for developing pre-eclampsia and preterm pre-eclampsia in women referred to a specialist compared with standard care was 5.5 and 12.2, respectively.

Conclusions The ability to predict pre-eclampsia in healthy nulliparous women using clinical phenotype is modest and requires external validation in other populations. If validated, it could provide a personalised clinical risk profile for nulliparous women to which biomarkers could be added.

Trial registration ACTRN12607000551493.

\section{INTRODUCTION}

Pre-eclampsia is a multisystem complication that occurs after 20 weeks of pregnancy and can cause considerable maternal and fetal morbidity and mortality. ${ }^{1}$ This complex condition is characterised by suboptimal uteroplacental perfusion associated with a maternal inflammatory response and maternal vascular endothelial dysfunction. ${ }^{2}$ One of the main reasons for serial clinical assessment in antenatal care is the early detection of signs (raised blood pressure and proteinuria) indicative of evolving pre-eclampsia. ${ }^{3}$ Recent guidelines from the National Institute for Health and Clinical Excellence (NICE) also recommend routine screening for specific risk factors for pre-eclampsia (nulliparity, older age, high body mass index (BMI), family history of pre-eclampsia, underlying renal disease or chronic hypertension, multiple pregnancy, more than 10 years between pregnancies, and a personal history of pre-eclampsia). ${ }^{3}$ The expected rate of pre-eclampsia when any one of these risk factors is present ranges from $3 \%$ to more than $30 \%$, and many women have several risk factors. ${ }^{4-7}$ The absolute risk for an individual will be determined by the presence or absence of these and other predisposing or protective factors not incorporated in the NICE 
guidelines. ${ }^{8-13}$ Currently, because of a paucity of large prospective studies, we cannot accurately estimate the risk of pre-eclampsia from combinations of clinical risk factors. ${ }^{414}$

In prospective studies of general obstetric populations, the reported performance of a limited number of clinical risk factors to predict pre-eclampsia is modest, with an AUC (area under the curve) in the order of 0.66 to $0.79 .^{1516}$ These cohorts included high risk women and the best predictors of pre-eclampsia (underlying medical conditions that predispose to preeclampsia or a history of pre-eclampsia ${ }^{4616}$ ) are not applicable to healthy nulliparous women. Before preventive treatment and stratified antenatal care can be offered to nulliparous women, we need to identify those at high risk of pre-eclampsia. ${ }^{1718}$ At present there is no method to accurately stratify healthy nulliparous women according to their risk profile for preeclampsia.

This study is part of the SCOPE (Screening for Pregnancy Endpoints) study, a prospective, multicentre cohort study of "healthy" nulliparous women with the primary aim of developing screening tests to predict pre-eclampsia, infants who are small for gestational age, and spontaneous preterm birth. The study design incorporates prospective collection of information on all known clinical risk factors for pre-eclampsia. The objectives are to develop multivariable predictive models for pre-eclampsia (based on clinical risk factors present in early pregnancy alone or in combination with ultrasound estimates of uteroplacental perfusion and fetal measurements at 19-21 weeks' gestation) and determine their performance to predict pre-eclampsia as a baseline for future external validation; identify the rate of pre-eclampsia associated with specific combinations of clinical risk factors and ultrasound scan variables; and develop a proposal for risk stratification of "healthy" nulliparous women, based on combinations of key clinical risk factors and scan indices, to identify a subgroup at increased risk of pre-eclampsia for whom specialist referral might be indicated.

\section{METHODS}

Five centres (Auckland, New Zealand; Adelaide, Australia; London and Manchester, UK; and Cork, Ireland) recruited nulliparous women with singleton pregnancies to the SCOPE study between November 2004 and August 2008. ${ }^{19}$

Women ( $\mathrm{n}=4961)$ attending hospital antenatal clinics, obstetricians, general practitioners, or community midwives before 15 weeks' gestation were invited to participate. Exclusion criteria included recognised as high risk of pre-eclampsia, small for gestational age baby or spontaneous preterm birth because of underlying medical conditions (chronic hypertension requiring antihypertensive drugs, diabetes, renal disease, systemic lupus erythematosus, antiphospholipid syndrome, sickle cell disease, HIV), previous cervical knife cone biopsy, three or more abortions or three or more miscarriages, current ruptured membranes; known major fetal anomaly or abnormal karyotype; or intervention that could modify the outcome of pregnancy (such as aspirin, cervical suture). ${ }^{19} \mathrm{~A}$ research midwife interviewed and examined women at 14-16 and 19-21 weeks' gestation. Women underwent an ultrasound scan at 19-21 weeks. At the time of interview, data were entered on an internet accessed central database with a complete audit trail (MedSciNet).

At 14-16 weeks' gestation the following data were collected: demographic information including age, ethnicity, immigration details, education, work, socioeconomic index, income level, living situation; the woman's birth weight and gestation at delivery and whether it was a singleton or multiple pregnancy; previous miscarriages, abortions, or ectopic pregnancies and whether these pregnancies were with the same partner as the current pregnancy or not; history of infertility, use of assisted reproductive technologies, duration of sexual relationship, and exposure to partner's sperm; gynaecological (including polycystic ovarian syndrome) and medical history, including hypertension while taking combined oral contraception, asthma, urinary tract infection, inflammatory bowel disease, thyroid disease, and thromboembolism; and family history (in mother and sisters) of obstetric complications (miscarriage, pre-eclampsia, eclampsia, gestational hypertension, spontaneous preterm birth, any preterm birth, gestational diabetes, stillbirth, and neonatal death) and family history (mother, father, sibling) of medical conditions (hypertension, coronary artery heart disease, cerebrovascular accident, type 1 and 2 diabetes, and venous thromboembolism).

Information was collected on vaginal bleeding early in pregnancy (gestation, severity and duration of bleeding, and recurrent bleeds), hyperemesis, and infections during pregnancy. Vegetarian status was recorded, and other dietary information before conception and during pregnancy was obtained from food frequency questions for fruit, green leafy vegetables, oily and other fish, and fast foods. Use of folate and multivitamins, cigarettes, alcohol (including binge drinking), and recreational drugs (including marijuana, amphetamine, cocaine, heroin, ecstasy, LSD (lysergide)) was recorded for before conception, first trimester, and at 15 weeks. A lifestyle questionnaire was completed on work, exercise and sedentary activities, snoring, domestic violence, and social supports. Psychological scales were completed to measure perceived stress, ${ }^{20}$ depression, ${ }^{21}$ anxiety, ${ }^{22}$ and behavioural responses to pregnancy (adapted from the behavioural responses to illness questionnaire ${ }^{23}$ ). Two consecutive manual blood pressure measurements (mercury or aneroid sphygmomanometer, with a large cuff if the arm circumference $\geq 33 \mathrm{~cm}$ and Korotkoff $\mathrm{V}$ for diastolic blood pressure) were recorded. Other maternal measurements included maternal height and weight and waist, hip, arm, and head circumference. Proteinuria in a midstream urine specimen was measured by dipstick or a protein:creatinine ratio. Random whole blood glucose and serum lipid concentrations (triglycerides, total cholesterol, high density lipoprotein cholesterol, low density lipoprotein cholesterol, total 
cholesterol:high density lipoprotein cholesterol ratio) were also measured.

Ultrasound examination at 19-21 weeks' gestation included measurements of the fetus (biparietal diameter, head circumference, abdominal circumference, and femur length) and Doppler studies of the umbilical and uterine arteries. ${ }^{24}$ All fetal measurements were adjusted for gestational age by calculating the multiple of the median for each gestational week. Mean uterine resistance index (RI) was calculated from the left and right uterine resistance index. If only a left or right uterine resistance index was available, this was used as "mean resistance index" ( $\mathrm{n}=20)$. Notching of each uterine artery was recorded. An abnormal uterine artery Doppler result was defined as a mean resistance index $>90$ th centile $(>0.695)$.

Participants were followed prospectively, and research midwives collected data on pregnancy outcome and measurements of the baby. Data monitoring included individual checks of all data for each participant, including checks for any transcription errors of the lifestyle questionnaire, and detection of illogical or inconsistent data and outliers with customised software.

\section{Primary outcome}

Our primary outcome was pre-eclampsia defined as systolic blood pressure $\geq 140 \mathrm{~mm} \mathrm{Hg}$ or diastolic blood pressure $\geq 90 \mathrm{~mm} \mathrm{Hg}$, or both, on at least two occasions four hours apart after 20 weeks' gestation but before the onset of labour, or postpartum, with either proteinuria (24 hour urinary protein $\geq 300 \mathrm{mg}$ or spot urine protein:creatinine ratio $\geq 30 \mathrm{mg} / \mathrm{mmol}$ creatinine or urine dipstick protein $\geq++$ ) or any multisystem complication of pre-eclampsia. ${ }^{1925}$ Multisystem complications included any of acute renal insufficiency defined as a new increase in serum creatinine concentration $\geq 100 \mu \mathrm{mol} / \mathrm{L}$ antepartum or $>130$ $\mu \mathrm{mol} / \mathrm{L}$ postpartum; effects on liver, defined as raised aspartate transaminase or alanine transaminase concentration, or both, $>45 \mathrm{IU} / \mathrm{L}$ and/or severe right upper quadrant or epigastric pain or liver rupture; neurological effects included eclampsia, imminent eclampsia (severe headache with hyper-reflexia and persistent visual disturbance), or cerebral haemorrhage; and haematological effects included thrombocytopenia (platelets $<100 \times 10^{9} / \mathrm{L}$ ), disseminated intravascular coagulation, or haemolysis. The reference group was women who did not develop preeclampsia.

\section{Other definitions}

The estimated date of delivery was calculated as follows: if the woman was certain of the date of her last menstrual period (LMP), the estimated date of delivery was adjusted only if a scan at $<16$ weeks' gestation found a difference of seven or more days between the scan gestation and that calculated by the LMP or a scan at $20 \pm 1$ weeks found a difference of 10 or more days. If her date was uncertain, scan dates were used to calculate the estimated date of delivery. Preterm pre-eclampsia was pre-eclampsia resulting in delivery before $37^{+0}$ weeks' gestation. Small for gestational age was defined as a birth weight below the 10th customised centile, adjusted for maternal height, booking weight, ethnicity, and delivery gestation and infant's sex. ${ }^{2627}$

\section{Power calculation}

The number of women required to be screened was based on achieving suitable screening test characteristics and precise estimates of their values. Given a pretest probability (prevalence) of pre-eclampsia of 5\%, then a post-test probability of $30 \%$ or greater would make this a useful test, based on current clinical practice. The algorithm must therefore have sufficient ability such that it is unlikely the post-test probability will fall below $0.30(30 \%)$ for pre-eclampsia. This can be attained, with a power of $80 \%$, in a cohort of 3000 if the true positive likelihood ratio of the screening test is 9.2 to 10.0 . Given a prevalence of $5 \%$, if we observe a sensitivity of $90 \%$ this cohort size will give a $95 \%$ confidence interval for this sensitivity of 84.0 to 94.3 , and a specificity of $91 \%$.

\section{Datasets}

We used two datasets to construct the predictive models for pre-eclampsia. The first comprised clinical variables obtained at 16-16 weeks' gestation and the second comprised clinical data at 14-16 weeks combined with variables from the 19-21 week ultrasound scan. Of the 933 original and derived variables recorded, we excluded variables added after recruitment commenced $(n=76)$, paternal variables $(n=48)$, variables not applicable to prediction of pre-eclampsia ( $n=246)$, variables with more than $10 \%$ missing data (clinical laboratory data and work variables not applicable to women not working, $\mathrm{n}=27$ ), and 402 variables with $\mathrm{P}>0.10$ on univariable comparison of women with and without pre-eclampsia. Of the remaining 134 variables, we selected 38 as candidate predictors on the following criteria: known potential risk factors for pre-eclampsia, ease of collection in the clinical setting, and potential applicability to future populations (see table A in appendix 1 on bmj.com for a full list of variables). With this approach the only established "risk" factor not included in the candidate predictors was cigarette smoking, as in our dataset this was not associated with pre-eclampsia. We added the number of cigarettes smoked a day at 15 weeks' gestation as a candidate predictor, giving a total of 39 variables for the multivariable analysis. Variables were not included as candidate predictors because of colinearity $(n=61)$, a low cell count $(<5)$ in the $\chi^{2}$ test $(n=11)$, lack of a consistent relation with pre-eclampsia in literature $(n=4)$, or not readily applicable to a future obstetric population $(\mathrm{n}=20)$.

Among the 39 candidate predictors, data were complete in 32 , missing in $<1 \%$ for six variables, and missing in $6 \%$ for participant's birth weight. We imputed missing continuous data $(n=4)$ with expectation maximisation and used the median for three variables unrelated to other observed data. The expectation maximisation algorithm was implemented in the 
Table 1|Characteristics of participants at 14-16 weeks' gestation by pre-eclampsia status. Figures are numbers (percentages) unless stated otherwise

\begin{tabular}{|c|c|c|c|}
\hline & $\begin{array}{l}\text { No pre-eclampsia } \\
(n=3343)\end{array}$ & $\begin{array}{l}\text { Pre-eclampsia } \\
\quad(n=186)\end{array}$ & Pvalue* \\
\hline Mean (SD) maternal age (years) & $28.2(5.8)$ & $27.0(5.8)$ & 0.004 \\
\hline \multicolumn{4}{|l|}{ Ethnicity: } \\
\hline White & $2903(87)$ & $158(85)$ & \multirow{4}{*}{0.50} \\
\hline Maori or Polynesian & $106(3)$ & $9(5)$ & \\
\hline Asian & $242(7)$ & $12(6)$ & \\
\hline Other & $92(3)$ & 7 (4) & \\
\hline Married/cohabiting & $3080(92)$ & $172(93)$ & 0.87 \\
\hline Mean (SD) socioeconomic index & $41(17)$ & $37(16)$ & $<0.001$ \\
\hline «12 years' schooling & $1581(47)$ & $98(53)$ & 0.15 \\
\hline Tertiary education & $2670(80)$ & $140(75)$ & 0.13 \\
\hline Full/part time work & $2798(84)$ & $148(80)$ & 0.16 \\
\hline Primigravida & $2484(74)$ & $138(74)$ & 0.97 \\
\hline Previous miscarriage & 487 (15) & $28(15)$ & 0.83 \\
\hline Previous termination & $463(14)$ & $22(12)$ & 0.51 \\
\hline \multicolumn{4}{|l|}{ Smoking status: } \\
\hline Non-smoker & $2600(78)$ & $146(78)$ & \multirow{3}{*}{0.81} \\
\hline Stopped during pregnancy & $371(11)$ & $22(12)$ & \\
\hline Current smokers & $372(11)$ & $18(10)$ & \\
\hline \multicolumn{4}{|l|}{ BMI: } \\
\hline$<20.0$ & $58(2)$ & $1(1)$ & \multirow{4}{*}{$<0.001$} \\
\hline $20.0-24.9$ & $1830(55)$ & $70(37)$ & \\
\hline $25.0-29.9$ & $936(28)$ & $55(30)$ & \\
\hline$\geq 30$ & $519(15)$ & $60(32)$ & \\
\hline \multicolumn{4}{|l|}{ Mean (SD) blood pressure $(\mathrm{mm} \mathrm{Hg})$ : } \\
\hline Systolic & $108(11)$ & $114(11)$ & $<0.001$ \\
\hline Diastolic & $64(8)$ & $69(8)$ & $<0.001$ \\
\hline
\end{tabular}

BMI=body mass index.

*For comparisons between groups with $x^{2}$ or Student's $t$ test.

"mix" package in $\mathrm{R}$, version 2.9.1. ${ }^{2829}$ To evaluate its imputation, we used a permutation technique on the complete dataset. For each variable, we systematically removed each data point and imputed the "missing" value using expectation maximisation. We calculated the ratio of mean absolute error between imputed and original values to the mean value for that variable. The mean ratio for the variables imputed with expectation maximisation was $10.8 \%$.

\section{Statistical methods}

We used SAS (version 9.1) for univariable data analysis and to generate a multivariable logistic regression model. We used Student's $t$ test, Wilcoxon rank sum test, or $\chi^{2}$ test for comparing characteristics in the study population and pregnancy outcomes between women who did and did not develop pre-eclampsia. Stepwise logistic regression was used to determine independent risk factors for pre-eclampsia in both datasets. The order of variable selection was determined by the $\chi^{2}$ statistic for each potential variable and the forward selection step could be followed by removal of variables in one or more backward elimination steps. We calculated receiver operating characteristics curves and determined screening test characteristics at a $25 \%, 10 \%$, and $5 \%$ false positive rate. For internal validation we evaluated the calibration and discrimination (10-fold cross validation) of the model using methods described by Altman et al. ${ }^{30}$ Calibration was assessed by plotting the observed proportion of events against the predicted probabilities. For the cross validation, participants were stratified by region (New Zealand, Australia, Ireland, and UK), pre-eclampsia status (positive or negative), and gestation ( $<260$ days or $\geq 260$ days) and randomly allocated to one of 10 groups. Tenfold cross validation was then performed, with $90 \%$ of the data used to generate a model, and estimation of disease risk was performed in the $10 \%$ remaining. These predicted values were then combined across the 10 runs and summarised by the $\mathrm{C}$ statistic (AUC). This entire procedure was repeated 10 times.

To determine the variables most consistently retained in the prediction models, we generated the 10 "best models," based on the proportion of variance explained, by calculating all possible logistic regression models retaining 10 variables. We determined the frequency of each variable present in the 10 highest scoring models and identified key risk factors. We then calculated the proportion of women with specific combinations of key clinical risk factors and abnormal uterine artery Doppler at 20 weeks' gestation who developed pre-eclampsia.

\section{RESULTS}

Of the nulliparous women $(\mathrm{n}=4961)$ invited to participate in the study, $3780(76 \%)$ agreed but a further 208 were excluded before or at the 15 week interview (fig 1). Among the 3572 women recruited into the study, we had data on pregnancy outcome for 3529 $(99 \%)$. When we compared the women who declined to participate $(\mathrm{n}=1202)$ with the 3529 women in the study population there were minor but significant differences in the ethnicity mix $(79 \% v 87 \%$ white, $4 \% v$ $3 \%$ Maori or Pacific Islander, 11\% v 7\% Asian, 6\% v 3\% other, $\mathrm{P}<0.001$ ) and age (mean 28.8 (SD 5.7) v28.1 (SD $5.8), \mathrm{P}=0.001)$. A further 182 women were excluded

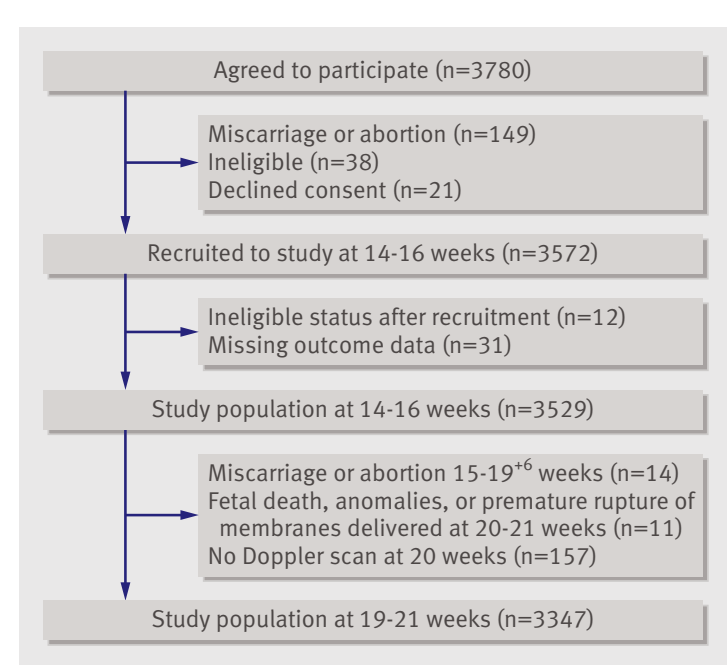

Fig 1 Recruitment and flow of participants through study 
Table 2 |Characteristics of pregnancy outcomes by pre-eclampsia status. Figures are numbers (percentages) unless stated otherwise

\begin{tabular}{|c|c|c|c|}
\hline & $\begin{array}{l}\text { No pre-eclampsia } \\
\quad(n=3343)\end{array}$ & $\begin{array}{l}\text { Pre-eclampsia } \\
\quad(n=186)\end{array}$ & P value* \\
\hline \multicolumn{4}{|l|}{ Maternal } \\
\hline \multicolumn{4}{|l|}{ Mean (SD) blood pressure $(\mathrm{mm} \mathrm{Hg})$ : } \\
\hline Systolic & $122(13)$ & $164(18)$ & $<0.001$ \\
\hline Diastolic & $75(10)$ & $104(10)$ & $<0.001$ \\
\hline \multicolumn{4}{|l|}{ Fetal } \\
\hline No of infants & $3329 \dagger$ & 186 & \\
\hline Mean (SD) birth weight (g) & $3408(581)$ & $3033(809)$ & $<0.001$ \\
\hline Mean (SD) gestation at delivery (weeks) & $39.5(2.7)$ & $37.9(2.8)$ & $<0.001$ \\
\hline SGA ( $(10$ th customised centile) $\ddagger$ & $332(10)$ & $44(24)$ & $<0.001$ \\
\hline \multicolumn{4}{|l|}{ Preterm birth: } \\
\hline «37 weeks & $214(6)$ & $47(25)$ & $<0.001$ \\
\hline «34 weeks & $76(2)$ & $19(10)$ & $<0.001$ \\
\hline Admission to neonatal unit & $349(11)$ & $57(31)$ & $<0.001$ \\
\hline Perinatal death & $18(0.5)$ & $1(0.5)$ & 1.0 \\
\hline \multicolumn{4}{|c|}{$\begin{array}{l}\text { SGA=small for gestational age. } \\
\text { *P values are comparisons between groups with } x^{2} \text { or Student's } t \text { test. } \\
\text { †Excludes miscarriages and abortions. } \\
\text { †For } 3327 \text { women in "no pre-eclampsia" group as customised centiles unavailable when fetus died in utero } \\
\text { before } 20 \text { weeks' gestation. }\end{array}$} \\
\hline
\end{tabular}

from the $20 \pm 1$ week dataset, in most cases because of missing data from the Doppler ultrasound $(\mathrm{n}=157)$ (fig 1).

In total $186(5 \%)$ women developed pre-eclampsia; in eight the diagnosis was postpartum and $47(1 \%)$ delivered preterm. Table 1 shows background characteristics and table 2 shows outcomes of pregnancy in women who did and did not develop pre-eclampsia. Women who developed pre-eclampsia were younger, had a lower socioeconomic index, and at 15 weeks' gestation were more likely to be obese and have higher blood pressure. Pre-eclampsia developed at a mean of 36.9 (SD 3.3) weeks' gestation, with a median protein: creatinine ratio of $88 \mathrm{mg} / \mathrm{mmol}$ (range $30-2445 \mathrm{mg}$ / $\mathrm{mmol}$ ) and 24 hour urinary protein excretion of $0.78 \mathrm{~g}$ (range $0.30-9.9 \mathrm{~g}$ ). The diagnosis of pre-eclampsia was based on hypertension in combination with multisystem complications in 24 of the 186 women $(13 \%)$, four of whom had "+" proteinuria. Forty two per cent of the women had at least one multisystem complication: $8 \%(n=14)$ had a diagnosis of HELLP (haemolysis, elevated liver enzymes, and low platelets) or ELLP (elevated liver enzymes and low platelets), 5\% $(n=9)$ developed impaired renal function, and one woman had eclampsia. A quarter of the babies were born preterm and $24 \%$ were small for gestational age.

Prediction of pre-eclampsia with clinical risk factors and uterine artery Doppler

Table 3 shows the clinical risk factors independently associated with pre-eclampsia on multivariable analysis (see tables B1 and B2 in appendix 2 on bmj.com for unadjusted odds ratios). Addition of ultrasound scan variables to the 15 week clinical data resulted in age and the number of cigarettes a day being removed from the model and inclusion of duration of sexual relationship of six months or less and uterine artery Doppler waveform indices. Based on clinical risk factors, the mean AUC from the ten 10-fold cross validations was 0.71 (SE 0.002) (fig 2). The AUC for the proposed model based on the observations used to create the model was 0.76 , indicating a bias in the $\mathrm{C}$ statistic of about $5 \%$. The addition of 20 week uterine artery Doppler indices did not improve performance based on the study population (internally validated AUC 0.71 (SE 0.003)). Figure 3 shows that the model has a reasonable level of calibration, but there is an indication that, at the higher probabilities for preeclampsia, it might underestimate cases.

Table 4 summarises the screening characteristics of the models at a false positive rate of $5 \%, 10 \%$, and $25 \%$ based on the women from whom the model was created and from the internal validation where the values reported are the means of those derived from each of the cross validation analyses.

To estimate a woman's probability of pre-eclampsia, a risk score can be calculated based on the formulas in the footnote of table 3 . The predicted probability of pre-eclampsia can then be calculated from $1 /\left(1+\mathrm{e}^{- \text {riskscore }}\right) \cdot{ }^{31}$ For example, for a 28 year old nulliparous woman whose birth weight was $2400 \mathrm{~g}$, with a mean arterial pressure of $96 \mathrm{~mm} \mathrm{Hg}, \mathrm{BMI} 30$, a family history of pre-eclampsia, and no protective factors, her probability of pre-eclampsia is $39 \%$. Her risk of pre-eclampsia decreases as each risk factor is removed in stepwise fashion; if her mean arterial pressure is $80 \mathrm{~mm} \mathrm{Hg}$ her probability of pre-eclampsia would be $18 \%$, if her BMI was 24 her probability would be $14 \%$, if she had no family history of preeclampsia her probability would be $8 \%$, and if her birth weight had been $3500 \mathrm{~g}$ her probability would be $5 \%$. If she had protective factors, such as a previous early miscarriage with her partner, her risk would be reduced to $2 \%$.

\section{Impact of definition of pre-eclampsia}

To evaluate the impact of 24 women receiving a diagnosis of pre-eclampsia based on the presence of gestational hypertension combined with multisystem complications, the model was reconstructed defining

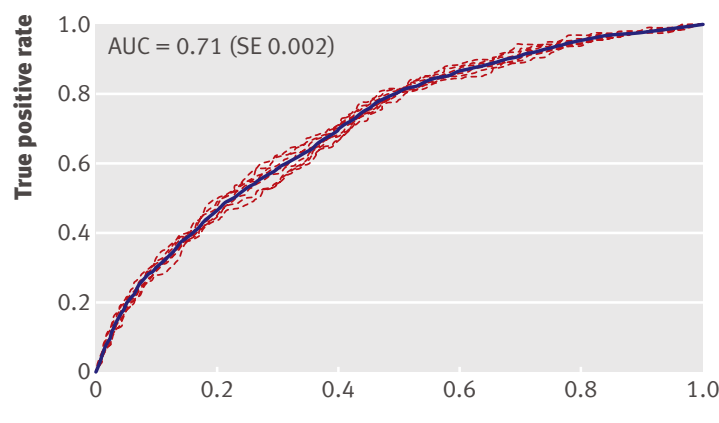

False positive rate

Fig 2 | Receiver operating characteristics curves based on independent predicted values from ten 10 -fold cross validation runs of model of clinical risk factors at 15 weeks 
Table 3 |Clinical risk factors at 15 weeks and ultrasound scan variables at 20 weeks included in logistic regression model for pre-eclampsia

\begin{tabular}{|c|c|c|c|c|c|c|}
\hline & \multicolumn{3}{|c|}{ Clinical risk factors at 15 weeks* } & \multicolumn{3}{|c|}{ Plus ultrasound scan at 20 weeks $\dagger$} \\
\hline & $\begin{array}{l}\text { No pre-eclampsia } \\
(n=3343)\end{array}$ & $\begin{array}{c}\text { Pre-eclampsia (n } \\
=186)\end{array}$ & $\begin{array}{l}\text { Adjusted OR } \\
\quad(95 \% \mathrm{Cl})\end{array}$ & $\begin{array}{l}\text { No pre-eclampsia } \\
(n=3167)\end{array}$ & $\begin{array}{l}\text { Pre-eclampsia } \\
\quad(n=180)\end{array}$ & $\begin{array}{l}\text { Adjusted OR } \\
(95 \% \mathrm{Cl})\end{array}$ \\
\hline \multicolumn{7}{|l|}{ Risk factors $\ddagger$} \\
\hline Decrease of 5 years in age & $28.2(5.8)$ & $27(5.8)$ & $1.2(1.1$ to 1.4$)$ & - & - & - \\
\hline Increase of $5 \mathrm{~mm} \mathrm{Hg}$ in MAP§ & $78(8)$ & $84(8)$ & $1.4(1.3$ to 1.5$)$ & $78(8)$ & $84(8)$ & $1.4(1.2$ to 1.5$)$ \\
\hline Increase of 5 in BMI & $25.4(5.1)$ & $28.2(6.7)$ & $1.3(1.1$ to 1.4$)$ & $25.4(5.1)$ & $28.3(6.8)$ & $1.3(1.1$ to 1.5$)$ \\
\hline Family history of pre-eclampsia & $309(9 \%)$ & $35(19 \%)$ & $2.0(1.3$ to 3.0$)$ & $299(9 \%)$ & $35(19 \%)$ & 1.9 (1.3 to 2.9$)$ \\
\hline Family history of coronary heart disease & $384(12 \%)$ & 35 (19\%) & 1.9 (1.2 to 2.8$)$ & $359(11 \%)$ & 35 (19\%) & 1.8 (1.2 to 2.8$)$ \\
\hline Decrease of $500 \mathrm{~g}$ in woman's birth weight** & $3293(552)$ & $3177(540)$ & $1.2(1.1$ to 1.4$)$ & $3292(551)$ & $3177(547)$ & $1.2(1.1$ to 1.4$)$ \\
\hline Vaginal bleeding $\geq 5$ days & $125(4 \%)$ & $13(7 \%)$ & 2.0 (1.1 to 3.8$)$ & $116(4 \%)$ & $12(7 \%)$ & 1.9 (1.0 to 3.7$)$ \\
\hline$\leq 6$ months in sexual relationship $†$ & - & - & - & $256(8 \%)$ & $26(14 \%)$ & $1.7(1.04$ to 2.6$)$ \\
\hline Bilateral notches & - & - & - & $327(10 \%)$ & $33(18 \%)$ & 1.7 (1.1 to 2.6$)$ \\
\hline Increase of 0.1 in mean uterine artery RI & - & - & - & $0.56(0.10)$ & $0.60(0.12)$ & $1.2(1.04$ to 1.5$)$ \\
\hline \multicolumn{7}{|l|}{ Protective factors } \\
\hline One miscarriage $\leq 10$ weeks, same partner & $301(9 \%)$ & $8(4 \%)$ & $0.45(0.22$ to 0.93$)$ & $284(9 \%)$ & $8(4 \%)$ & $0.44(0.21$ to 0.92$)$ \\
\hline$\geq 12$ months to conceive & $384(12 \%)$ & $12(7 \%)$ & $0.40(0.22$ to 0.75$)$ & $357(11 \%)$ & $12(7 \%)$ & $0.41(0.22$ to 0.76$)$ \\
\hline High fruit intake ( $\geq 3 /$ day) & $1347(40 \%)$ & $52(28 \%)$ & $0.69(0.49$ to 0.98$)$ & $1266(40 \%)$ & $48(27 \%)$ & 0.65 (0.45 to 0.92$)$ \\
\hline Alcohol consumption 1st trimester & $1705(51 \%)$ & $69(37 \%)$ & $0.60(0.44$ to 0.83$)$ & $1616(51 \%)$ & $65(36 \%)$ & 0.57 (0.41 to 0.79$)$ \\
\hline Increase of 5 cigarettes/day & $0.8(3.0)$ & $0.6(2.4)$ & $0.73(0.53$ to 1.0$)$ & - & - & - \\
\hline \multicolumn{7}{|c|}{ 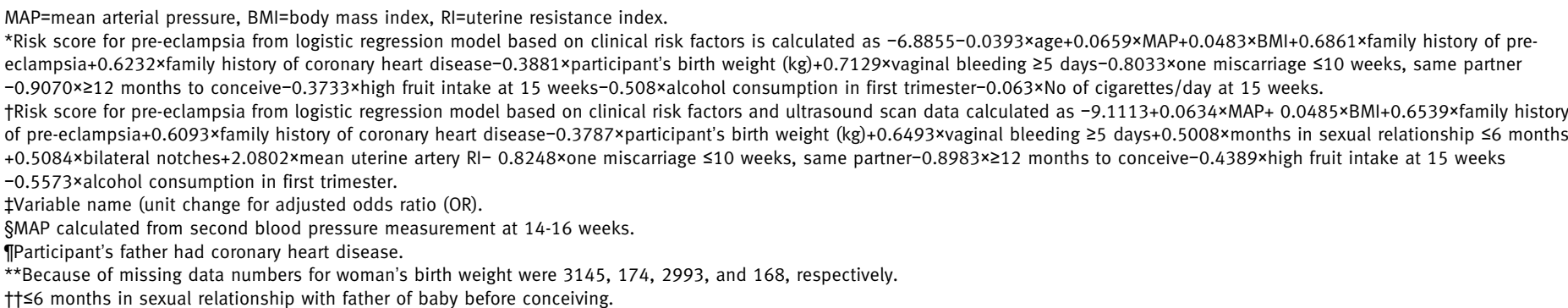 } \\
\hline
\end{tabular}

the cases as pre-eclamptic women with proteinuria $(\mathrm{n}=162)$. Most risk factors and protective factors remained with similar odds ratios, except that age, high intake of fruit, and cigarettes were excluded and a sexual relationship of six months or less (odds ratio $1.7,95 \%$ confidence interval 1.05 to 2.7$)$, hyperemesis at 15 weeks $(2.0,1.1$ to 3.7$)$, and maternal height $(0.87$, 0.76 to 1.0$)$ per $5 \mathrm{~cm}$ increase) were included.

\section{Reproducibility of prediction model}

To investigate the stability and potential reproducibility of the model (using all candidate predictors) we constructed 10 "best models" that included 10 variables. The risk factors (mean arterial blood pressure, BMI, family history of pre-eclampsia, family history of coronary heart disease (woman's father), participant's birth weight) and protective factors ( $\geq 12$ months to conceive, alcohol used in the first trimester) occurred in all " 10 best models." Of the other variables in our model (table 3), six occurred in three to seven of the best models, while cigarettes a day was not selected by the stepwise model fitting procedure.

Risk estimates with specific combinations of clinical risk factors

Table 5 shows the proportion of women with specific combinations of key clinical risk factors and abnormal result on uterine artery Doppler who developed preeclampsia. We have shown systolic blood pressure rather than mean arterial blood pressure as that requires calculation and, unless incorporated into an algorithm, is not easily applied in a routine clinic setting.

Specialist referral framework for nulliparous women To better understand potential clinical implications, we developed a framework for specialist referral in a population $(\mathrm{n}=1000)$ based on observations in our study population (fig 4 ). In the first stage, women

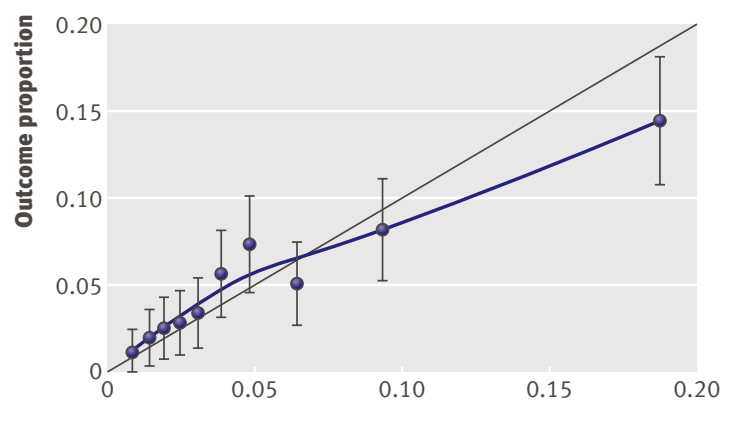

Predicted probability

Fig 3 | Observed rate of pre-eclampsia compared with predicted probabilities of pre-eclampsia based on clinical risk factors model 
Table 4|Prediction of pre-eclampsia from algorithms based on clinical risk factors at 14-16 weeks' gestation in cohort and independent predicted values from ten 10 -fold cross validation runs. Values are shown with $95 \%$ confidence intervals

\begin{tabular}{|c|c|c|c|c|c|c|}
\hline No (\%) with abnormal test result & Sensitivity & Specificity & $\begin{array}{l}\text { Positive predictive } \\
\text { value }\end{array}$ & $\begin{array}{l}\text { Negative predictive } \\
\text { value }\end{array}$ & Positive likelihood ratio & Negative likelihood ratio \\
\hline \multicolumn{7}{|l|}{ Training cohort* } \\
\hline $218(6)$ & 27 (22 to 34$)$ & 95 (94 to 96) & 24 (22 to 25$)$ & 96 (95 to 97$)$ & $5.5(4.2$ to 7.2$)$ & $0.76(0.70$ to 0.84$)$ \\
\hline $403(11)$ & 37 (30 to 44) & 90 (89 to 91) & 17 (16 to 18$)$ & 96 (96 to 97$)$ & $3.6(2.9$ to 4.5$)$ & 0.71 (0.63 to 0.79$)$ \\
\hline $949(27)$ & 61 (54 to 68$)$ & 75 (74 to 76$)$ & 12 (11 to 13$)$ & 97 (97 to 98) & 2.5 (2.2 to 2.8$)$ & $0.52(0.43$ to 0.62$)$ \\
\hline \multicolumn{7}{|l|}{ Ten 10 -fold cross validation $\dagger$} \\
\hline $205(6)$ & 20 (18 to 22$)$ & 95 (95 to 95) & 18 (16 to 20$)$ & 96 (95 to 96$)$ & 3.9 (3.5 to 4.4$)$ & 0.85 (0.83 to 0.87$)$ \\
\hline $392(11)$ & 30 (28 to 33$)$ & 90 (89 to 91$)$ & $14(13$ to 16$)$ & 96 (96 to 96$)$ & $3.0(2.8$ to 3.3$)$ & $0.78(0.75$ to 0.80$)$ \\
\hline $935(27)$ & 53 (48 to 58$)$ & 75 (74 to 76$)$ & 10 (10 to 12$)$ & 97 (96 to 97) & 2.1 (1.9 to 2.3 ) & $0.63(0.56$ to 0.70$)$ \\
\hline
\end{tabular}

*Based on clinical risk factor algorithm from table 2 using cut-offs at $5 \%, 10 \%$, and $25 \%$ false positive rates

†Average test characteristics across 10 different cross validation repeats.

were referred to a specialist if their post-test probability of pre-eclampsia generated by the model was at least $15 \%$. Among those not referred, women with a systolic blood pressure $>120 \mathrm{~mm} \mathrm{Hg}$, a BMI $\geq 30$, or a family history of pre-eclampsia underwent a uterine Doppler ultrasound with their fetal anatomy scan at 20 weeks, and those with an abnormal uterine artery resistance index also had a specialist referral. Of the women referred for a specialist opinion, $21 \%$ developed preeclampsia and $8 \%$ developed preterm pre-eclampsia. The relative risk for developing pre-eclampsia and preterm pre-eclampsia in the specialist referred group compared with standard care group was 5.5 and 12.2 , respectively.

Overall, application of this framework for specialist referral would result in $34 \%$ of women with preeclampsia (63 of 186) and 53\% of those who develop preterm pre-eclampsia (25 of 47) being referred. If the referral criteria did not include uterine Doppler, fewer cases of preterm pre-eclampsia (16 of 47, 34\%) would be detected. These results also show that negative prediction based on clinical risk assessment, with or without Doppler ultrasonography, is too inaccurate to allow a reduction in antenatal care.

\section{DISCUSSION}

In this large prospective international cohort of "healthy" nulliparous women an algorithm for preeclampsia, which included clinical risk factors at 15 weeks' gestation, had moderate predictive performance. The algorithm included well recognised risk factors (blood pressure, BMI, and a family history of pre-eclampsia) along with less established factors, such as prolonged vaginal bleeding, maternal low birth weight, and the woman's father having coronary artery disease. The algorithm had moderate predictive performance - the area under the receiver operating characteristics curve (AUC) was 0.76-and detected 37\% and $61 \%$ of women who developed pre-eclampsia with a false positive rate of $10 \%$ and $25 \%$, respectively. Addition of information from ultrasonography did not significantly improve performance of the algorithm, with an AUC of 0.77. We would expect poorer screening performance of the algorithm in other nulliparous populations, as evident by the AUC of 0.71 on internal validation. Given the prospective design, cohort size, comprehensive range of candidate predictors, high quality data, and completeness of follow-up, this is likely to be indicative of the best performance achievable using clinical and ultrasonography data to predict pre-eclampsia in a "healthy" nulliparous population. To considerably improve prediction performance will require either the development of specific clinical risk algorithms for disease subtypes, such as preterm and term pre-eclampsia, or the addition of biomarkers, or both.

The concept of a personalised clinical risk estimate for disease, to which biomarkers can be added, is established in several areas of medicine. The algorithm to predict pre-eclampsia reported here provides a first step towards a personalised risk score for pre-eclampsia among nulliparous women. It is inevitable the model will be overfitted to our population and external validation of the algorithm in other nulliparous populations is essential. We plan to evaluate its performance in the next 3000 women recruited into SCOPE, nearly all of whom will be recruited in different centres than the initial 3500 women. Validation should also be performed in other study populations of nulliparous women.

\section{Strengths and weaknesses}

A major strength of this study is its large multicentre prospective design with excellent follow-up. As the focus of the SCOPE study is development of tests to predict pregnancy outcome with potential to translate into clinical care, we recruited a clearly defined population of nulliparous women, enabling identification of similar populations for external validation. This is critical for generalisability of a risk assessment algorithm; the population in which the algorithm is developed needs to be identifiable if a screening test is to be used in clinical care. ${ }^{32}$

We obtained high quality data for all known risk factors for pre-eclampsia from questionnaires administered at interviews, along with detailed standard operating procedures. Use of a real time database, with automated checking procedures, reduced data entry errors and transcription errors. For a dataset of this size, the rate of missing data was minimal. The 
Table 5 |Probability of pre-eclampsia in presence of combinations of clinical risk factors at 15 weeks ${ }^{\star}$ and uterine artery Doppler at 20 weeks $\dagger$

\begin{tabular}{|c|c|c|c|c|c|c|c|c|c|}
\hline No of women & $\begin{array}{l}\text { Systolic BP } \\
>120 \mathrm{~mm} \mathrm{Hg}\end{array}$ & $\mathrm{BMI} \geq 30$ & FH PE† & $\begin{array}{l}\text { FH coronary } \\
\text { artery disease }\end{array}$ & $\begin{array}{l}\text { Maternal birth } \\
\text { weight }<2500 \mathrm{~g}\end{array}$ & $\begin{array}{l}\text { Vaginal bleeding } \\
\geq 5 \text { days }\end{array}$ & $\begin{array}{l}\text { Abnormal mean } \\
\text { uterine artery RI }\end{array}$ & $\begin{array}{l}\text { Proportion PE \% } \\
\quad(95 \% \mathrm{Cl})\end{array}$ & $\begin{array}{l}\text { Proportion } \\
\text { preterm PE \% } \\
(95 \% \mathrm{Cl})\end{array}$ \\
\hline 310 & $\bullet$ & - & - & - & - & - & - & 14 (10 to 18$)$ & 5 (3 to 8$)$ \\
\hline 579 & - & - & - & - & - & - & - & 10 (8 to 13$)$ & 2 (1 to 4$)$ \\
\hline 344 & - & - & - & - & - & - & - & 10 (7 to 14$)$ & 3 (2 to 6$)$ \\
\hline 419 & - & - & - & - & - & - & - & 8 (6 to 11$)$ & $3(2$ to 5$)$ \\
\hline 224 & - & - & - & - & - & - & - & 9 (6 to 13$)$ & 3 (2 to 6$)$ \\
\hline 138 & - & - & - & - & - & - & - & $9(6$ to 16$)$ & 1 (0.4 to 5$)$ \\
\hline 131 & - & - & - & - & - & - & - & 16 (11 to 23$)$ & 4 (2 to 9$)$ \\
\hline 46 & - & - & - & - & - & - & - & 20 (11 to 33$)$ & 7 (2 to 18$)$ \\
\hline 52 & - & - & - & - & - & - & - & 15 (8 to 28$)$ & 8 (3 to 18$)$ \\
\hline 21 & - & - & - & - & - & - & - & $33(17$ to 55$)$ & 24 (11 to 45$)$ \\
\hline 15 & - & - & - & - & - & - & - & 27 (11 to 52$)$ & 13 (4 to 38 ) \\
\hline 73 & - & - & - & - & - & - & - & 18 (11 to 28$)$ & 7 (3 to 15$)$ \\
\hline 77 & - & - & - & - & - & - & - & 14 (8 to 24$)$ & 4 (1 to 11$)$ \\
\hline 41 & - & - & - & - & - & - & - & 17 (9 to 31 ) & 5 (1 to 16$)$ \\
\hline 24 & - & - & - & - & - & - & - & 8 (2 to 26$)$ & - \\
\hline 12 & - & - & - & - & - & - & - & 42 (19 to 68$)$ & - \\
\hline 24 & - & - & - & - & - & - & - & 17 (7 to 36$)$ & 8 (2 to 26$)$ \\
\hline 326 & - & - & - & - & - & - & $\bullet$ & 10 (7 to 14$)$ & 6 (4 to 9 ) \\
\hline 29 & - & - & - & - & - & - & - & 24 (12 to 42$)$ & 14 (6 to 31$)$ \\
\hline 65 & - & - & - & - & - & - & - & 17 (10 to 28$)$ & 11 (5 to 21$)$ \\
\hline 44 & - & - & $\bullet$ & - & - & - & $\bullet$ & 16 (8 to 29$)$ & 16 (8 to 29$)$ \\
\hline 32 & - & - & - & - & - & - & - & 13 (5 to 28 ) & 6 (2 to 20$)$ \\
\hline 25 & - & - & - & - & - & - & - & 8 (2 to 25$)$ & - \\
\hline 33 & - & - & - & - & - & - & - & 9 (3 to 24$)$ & 9 (3 to 24$)$ \\
\hline
\end{tabular}

$\mathrm{BP}=$ blood pressure; $\mathrm{FH}=$ family history; $\mathrm{PE}=$ pre-eclampsia; $\mathrm{RI}=$ resistance index.

*In 3529 women; $5 \%$ developed pre-eclampsia and $1 \%$ developed preterm pre-eclampsia.

†In 3347 women; abnormal result=uterine artery RI $>0.695$ or bilateral notches.

intensive two stage data monitoring adds confidence in data integrity. Potential measurement errors, such as in self reported family history, ${ }^{33}$ could have occurred, but as the goal was to develop a prototype algorithm ultimately for clinical use, this limitation was accepted. Principal investigators reviewed outcome data for cases, ensuring accurate diagnosis. One of the challenges when predicting rare events in prospective cohorts, such as SCOPE, is the relatively low numbers of cases compared with studies based on huge epidemiological databases. While the latter might have a substantially greater number of events, their interpretation is restrained by less accurate diagnosis.

There is no consensus as to the best method for selection of variables. ${ }^{31}$ Given the rich dataset of potential predictors for pre-eclampsia, we used a pruning step based on significance testing and then selected a subset of candidate variables on a priori knowledge. This could have introduced variable selection bias, but it is reassuring that the clinical risk factors and their strength of association with pre-eclampsia are consistent with the literature. While we could undertake only internal validation at this stage, external validation is planned.

\section{Comparisons with other studies}

Previous studies investigating risk factors for preeclampsia have used birth registries or hospital databases, ${ }^{634}$ randomised trials with negative results (that is, no treatment effect shown), ${ }^{3536}$ and, in a few studies, prospective cohorts (usually general obstetric populations) designed to investigate outcomes of pregnancy. ${ }^{912}{ }^{1337}$ Consistent with other contemporary studies, the women who developed pre-eclampsia were younger, more obese, and more likely to have lower socioeconomic status. ${ }^{37-39}$

Many of the risk factors included in the algorithm presented here are associated with a similar degree of risk to that previously reported, giving confidence regarding the potential applicability of the algorithm to other populations. Higher blood pressure within the normal range, a higher BMI, and a family history of pre-eclampsia had similar predictive characteristics to those observed in other studies..$^{89-41}$ In our algorithm, blood pressure was the most important risk factor driving the estimated probability of pre-eclampsia. ${ }^{9}$ Mean arterial pressure, rather than systolic or diastolic blood pressure blood, was selected by stepwise logistic regression and included in the model. ${ }^{8}$ If it was implemented into clinical practice, the clinician would derive the mean arterial pressure from systolic and diastolic blood pressure entered into the algorithm. A history of coronary artery disease in the woman's father was associated with a 1.9-fold increase in the risk of preeclampsia, consistent with a previous report and the association between pre-eclampsia and subsequent 
ischaemic heart disease. ${ }^{4243}$ Confirming the results of a case-control study, ${ }^{44}$ a lower maternal birth weight was associated with an increased risk of pre-eclampsia, with an even greater risk when low maternal birth weight coexisted with other key risk factors. Prolonged vaginal bleeding in early pregnancy was associated with a twofold increase in risk of pre-eclampsia. As reported by others, most of these bleeds were mild in severity, suggesting that a discrete bleeding pattern could be associated with later pre-eclampsia. ${ }^{45}$

Several factors were associated with a reduced risk of pre-eclampsia. A single early miscarriage with the same partner, eating a lot of fruit, and smoking were protective, again reassuringly consistent with previous reports. ${ }^{101246}$ The protective influence of cigarette smoking in our cohort was less than previously reported, and cigarettes did not remain in the model when we added uterine artery Doppler indices. ${ }^{46}$ Alcohol use in the first trimester was protective but requires confirmation in other cohorts. ${ }^{47}$ Obese women are reported to drink less alcohol, possibly because food fulfils their addictive behaviour. ${ }^{4849}$ Obesity is unlikely to be the only explanation, however, as the protective effect of alcohol is retained with BMI in the model, and there was no interaction between BMI and alcohol.

A recent series of publications reported algorithms to predict pre-eclampsia based on clinical risk factors in a general population comprising high risk women (previous pre-eclampsia and medical conditions), nulliparous women, and low risk women (multiparas with previous uncomplicated pregnancies). ${ }^{93650}$ A model is fitted to the population in which it was developed, using the available candidate predictors. ${ }^{30} \mathrm{~A}$ general antenatal population constructed of subgroups with different risk profiles is difficult to replicate and future "general populations" are likely to comprise a different case-mix. The importance of population differences is evident in the failure of one proposed algorithm to validate in a high risk population, ${ }^{51}$ raising questions as to more general applicability to other populations such as "healthy" nulliparous women. Poor performance on

Nulliparous women $(n=1000,5.3 \%$ develop pre-eclampsia and $1.3 \%$ preterm pre-eclampsia)

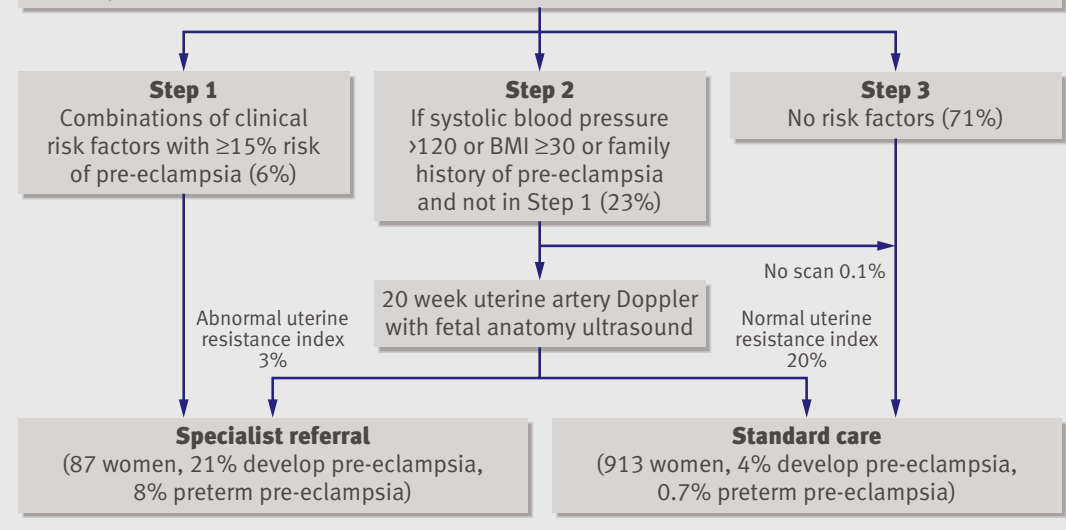

Fig 4 Framework for specialist referral when estimated risk of pre-eclampsia is $\geq 15 \%$ in model or presence of clinical risk factor with abnormal result on uterine artery Doppler scan validation might also occur because key predictors are missing from the model. When the list of candidate predictors includes strongly predictive factors, such as previous pre-eclampsia, renal disease, and chronic hypertension, ${ }^{1634}$ these will take precedence, replacing other factors that might be more relevant to healthy nulliparous women. In contrast, in SCOPE, we investigated candidate predictors applicable to healthy nulliparous women.

\section{Clinical relevance}

The new information on the rate of pre-eclampsia in the presence of combinations of specific risk factors (table 5) could be used by clinicians to improve current guidelines for specialist referral in nulliparous women. When we applied the criteria proposed in the NICE guidelines to the SCOPE cohort, $16.5 \%$ of nulliparous women would be referred for a specialist opinion of whom 10\% would develop pre-eclampsia. ${ }^{52}$ This included $31 \%$ of the 186 women who developed preeclampsia and $38 \%$ of the 47 of those who developed preterm pre-eclampsia. If we included only first pregnancy, as in the NICE guidelines, ${ }^{52} 12 \%$ would be referred and 23\% and 28\% cases of pre-eclampsia and preterm pre-eclampsia, respectively, would be detected. Our proposed framework for specialist referral based on the algorithm, along with uterine artery Doppler screening of a subpopulation, performed better than the NICE guidelines but requires validation. Among the referred women (9\% of nulliparas), the rate of pre-eclampsia was $21 \%$. Thirty four per cent of cases of pre-eclampsia and 53\% of cases of preterm preeclampsia were identified. This framework has the potential to identify a subgroup of nulliparous women at high risk of pre-eclampsia who could benefit from low dose aspirin and more intensive antenatal surveillance. It does not, however, provide additional information for the rest, whose risk is similar to an unscreened nulliparous population. Hence a negative "test" result would not modify current clinical care. The algorithm requires external validation, followed by assessment of the impact of increased surveillance, the false positive and false negative results, and a health economics analysis. If externally validated, this algorithm could help to inform future NICE guidelines for specialist referral. It could be made accessible, including via the web, as a support for risk stratification of healthy nulliparous women in low resource settings. To improve overall accuracy and detection of cases, the clinical algorithm will require the addition of biomarkers.

\section{Conclusions}

We have identified the most important clinical risk factors for pre-eclampsia in healthy nulliparous women and provided new information on the level of risk associated with specific combinations of risk factors. The predictive performance of the algorithm is modest, but offers a considerable improvement on current practice in healthy nulliparous women. As all known risk factors were included in this large prospective 


\section{WHAT IS ALREADY KNOWN ON THIS TOPIC}

There are many recognised clinical risk factors for pre-eclampsia, but the risk in nulliparous women associated with combinations of risk factors is largely unknown

No method exists to accurately risk stratify healthy nulliparous women

\section{WHAT THIS STUDY ADDS}

Use of data from a large international prospective cohort study provides new information on the level of risk of pre-eclampsia associated with specific combinations of clinical risk factors

The prototype algorithm based on clinical risk factors has modest ability to predict preeclampsia

If validated, the algorithm could provide a personalised estimation of clinical risk for preeclampsia in healthy nulliparous women to which biomarkers can be added

cohort, it shows the expected performance and limitations of using clinical phenotype to predict pre-eclampsia. The algorithm serves as a prototype that requires validation in other nulliparous populations. If validated, it might provide a personalised clinical risk profile for nulliparous women to which biomarkers could be added.

We thank the pregnant women who participated in the SCOPE study, Claire Roberts for her contributions in establishing the SCOPE study in Adelaide, Denise Healy for coordinating the Australian SCOPE study, Annette Briley for coordinating the UK MAPS (SCOPE) study, Nicolai Murphy for coordinating the Cork SCOPE study, the SCOPE research midwives, and Steven Wu for his assistance with data imputation. Contributors: RAN was responsible for conception and design, analysis and interpretation of data, and drafting the article and revising it critically for important intellectual content. LMEMCC, GAD, LP, JJW, and PNB were responsible for conception and design and interpretation of data, and critical revision of paper for important intellectual content. EHYC, AWS, and $M A B$ were responsible for statistical analyses and interpretation of data, and revising the article critically for important statistical content. RST was responsible for study design, coordination of clinical study, and revising the article critically for important intellectual content. LCK was responsible for conception and design, interpretation of data, drafting the article, and critical revision of paper for important intellectual content. Al authors had full access to all of the data (including statistical reports and tables) in the study, can take responsibility for the integrity of the data and the accuracy of the data analysis, and approved the final version to be published. RAN is guarantor.

Funding: This study was funded by New Enterprise Research Fund, Foundation for Research Science and Technology; Health Research Council (04/198); Evelyn Bond Fund, Auckland District Health Board Charitable Trust; Premier's Science and Research Fund, South Australian Government; Guy's and St Thomas' Charity, Tommy's the baby Charity; Biotechnology and Biological Sciences Research Council (GT084), UK National Health Services (NEAT grant FSD025), University of Manchester Proof of Concept Funding, NIHR; Health Research Board, Ireland (CSA/ 2007/2). The study sponsors had no role in study design, data analysis or writing this report. MAB received consultancy fees from the SCO PE Study, University of Auckland, which were funded by the New Zealand Health Research Council and the New Enterprise Research Fund, Foundation for Research Science and Technology.

Competing interests: All authors have completed the Unified Competing Interest form at www.icmje.org/coi_disclosure.pdf (available on request from the corresponding author) and declare: no support from any organisation for the submitted work; RAN and PNB have had consultancy relationships with Pronota in the previous three years; RAN has a consultancy relationship with Alere; LCK and PNB declare a US Provisional Patent Application in the name of University College Cork, Ireland (Louise Kenny and Philip Baker) "Detection of risk of preeclampsia" Application No USSN 61/288,465; RAN and MAB declares the following patent, which to date has not been licensed to a company: Blumenstein M, North RA, McMaster MT, Black MA, Kasabov NK, Cooper GIS. Biomarkers for prediction of pre-eclampsia and/or cardiovascular disease, PCT number WO/2009/108073. LP has a consultancy relationship with Tate and Lyle Research Advisory Group and is chairing a working party with ILSI Europe; both are outside the area of the submitted work.

Ethical approval: This study was approved by local ethics committees (New Zealand AKX/02/00/364, Australia REC 1712/5/2008, London and Manchester 06/MRE01/98, and Cork ECM5 (10) 05/02/08), and al women provided written informed consent.

Data sharing: No additional data available.

1 Sibai B, Dekker G, Kupferminc M. Pre-eclampsia. Lancet 2005;365:785-99.

2 Redman CW, Sargent IL. Latest advances in understanding preeclampsia. Science 2005;308:1592-4.

3 National Institute for Health and Clinical Excellence. NICE clinical guideline 62 . Antenatal care: routine care for the healthy pregnant woman. NICE, 2010.

4 Duckitt K, Harrington D. Risk factors for pre-eclampsia at antenatal booking: systematic review of controlled studies. $B M J$ 2005;330:565-7.

5 Chappell LC, Enye S, Seed P, Briley AL, Poston L, Shennan AH. Adverse perinatal outcomes and risk factors for preeclampsia in women with chronic hypertension: a prospective study. Hypertension 2008;51:1002-9.

6 Hernandez-Diaz S, Toh S, Cnattingius S. Risk of pre-eclampsia in first and subsequent pregnancies: prospective cohort study. $B M J$ 2009;338:b2255.

7 Sibai BM, Lindheimer M, Hauth J, Caritis S, VanDorsten P, Klebanoff M, et al. Risk factors for preeclampsia, abruptio placentae, and adverse neonatal outcomes among women with chronic hypertension. National Institute of Child Health and Human Development Network of Maternal-Fetal Medicine Units. N Engl/ Med 1998;339:667-71.

8 Cnossen JS, Vollebregt KC, de Vrieze N, ter Riet G, Mol BW, Franx A, et al. Accuracy of mean arterial pressure and blood pressure measurements in predicting pre-eclampsia: systematic review and meta-analysis. BMJ 2008;336:1117-20.

9 Poon LC, Kametas NA, Pandeva I, Valencia C, Nicolaides KH. Mean arterial pressure at $11(+0)$ to $13(+6)$ weeks in the prediction of preeclampsia. Hypertension 2008;51:1027-33.

10 Saftlas AF, Levine RJ, Klebanoff MA, Martz KL, Ewell MG, Morris CD, et al. Abortion, changed paternity, and risk of preeclampsia in nulliparous women. Am J Epidemiol 2003;157:1108-14

11 Trogstad L, Magnus P, Moffett A, Stoltenberg C. The effect of recurrent miscarriage and infertility on the risk of pre-eclampsia. BJOG 2009;116:108-13.

12 Brantsaeter AL, Haugen M, Samuelsen SO, Torjusen H, Trogstad L, Alexander J, et al. A dietary pattern characterized by high intake of vegetables, fruits, and vegetable oils is associated with reduced ris of preeclampsia in nulliparous pregnant Norwegian women. / Nutr 2009;139:1162-8

13 Ness RB, Markovic N, Bass D, Harger G, Roberts JM. Family history of hypertension, heart disease, and stroke among women who develop hypertension in pregnancy. Obstet Gynecol 2003;102:1366-71.

14 Milne F, Redman C, Walker J, Baker P, Bradley J, Cooper C, et al. The pre-eclampsia community guideline (PRECOG): how to screen for and detect onset of pre-eclampsia in the community. BMJ 2005;330:576-80

15 Papageorghiou AT, Yu CK, Erasmus IE, Cuckle HS, Nicolaides KH. Assessment of risk for the development of pre-eclampsia by maternal characteristics and uterine artery Doppler. BJOG 2005;112:703-9.

16 Poon LC, Kametas NA, Chelemen T, Leal A, Nicolaides KH. Materna risk factors for hypertensive disorders in pregnancy: a multivariate approach. J Hum Hypertens 2010;24:104-10.

17 Duley L, Henderson-Smart DJ, Meher S, King JF. Antiplatelet agents for preventing pre-eclampsia and its complications. Cochrane Database Syst Rev 2007;2:CD004659.

18 Hofmeyr GJ, Lawrie TA, Atallah AN, Duley L. Calcium supplementation during pregnancy for preventing hypertensive disorders and related problems. Cochrane Database Syst Rev 2010;8:CD001059.

19 McCowan L, North R, Taylor R. ACTRN12607000551493. Australian New Zealand Clinical Trials Registry. 2007. www.anzctr.org.au/ trialSearch.aspx.

20 Cohen S, Kamarck T, Mermelstein R. A global measure of perceived stress. J Health Soc Behav 1983;24:385-96.

21 Peindl KS, Wisner KL, Hanusa BH. Identifying depression in the first postpartum year: guidelines for office-based screening and referral. Affect Disord 2004:80:37-44.

22 Marteau TM, Bekker $\mathrm{H}$. The development of a six-item short-form of the state scale of the Spielberger State-Trait Anxiety Inventory (STAl). BrJ Clin Psychol 1992;31:301-6.

23 Spence M, Moss-Morris R, Chandler T. The behavioural responses to illness questionnaire (BRIO): a new predictive measure of medically 
unexplained symptoms following acute infection. Psychol Med 2005;35:583-93.

24 Groom KM, North RA, Stone PR, Chan EH, Taylor RS, Dekker GA, et al. Patterns of change in uterine artery Doppler studies between 20 and 24 weeks of gestation and pregnancy outcomes. Obstet Gynecol 2009;113:332-8.

25 Brown MA, Hague WM, Higgins J, Lowe S, McCowan L, Oats J, et al. The detection, investigation and management of hypertension in pregnancy: full consensus statement. Aust N ZJ Obstet Gynaecol 2000;40:139-55.

26 McCowan L, Stewart AW, Francis A, Gardosi J. A customised birthweight centile calculator developed for a New Zealand population. Aust N Z J Obstet Gynaecol 2004;44:428-31.

27 Gardosi J. Gestation network. www.gestation.net.

28 Schafer JL. Analysis of incomplete multivariate data. Chapman and Hall, 1996.

29 R Development Core Team. R: a language and environment for statistical computing. R Foundation for Statistical Computing,2009.

30 Altman DG, Vergouwe Y, Royston P, Moons KG. Prognosis and prognostic research: validating a prognostic model. $B M J$ 2009;338:b605.

31 Royston P, Moons KG, Altman DG, Vergouwe Y. Prognosis and prognostic research: developing a prognostic model. BMJ 2009;338:b604.

32 Moons KG, Altman DG, Vergouwe Y, Royston P. Prognosis and prognostic research: application and impact of prognostic models in clinical practice. BMJ 2009;338:b606.

33 Klemmensen AK, Olsen SF, Osterdal ML, Tabor A. Validity of preeclampsia-related diagnoses recorded in a national hospital registry and in a postpartum interview of the women. Am J Epidemiol 2007; 166:117-24

34 Mostello D, Catlin TK, Roman L, Holcomb WL Jr, Leet T. Preeclampsia in the parous woman: who is at risk? Am J Obstet Gynecol 2002;187:425-9.

35 Sibai BM, Ewell M, Levine RJ, Klebanoff MA, Esterlitz J, Catalano PM, et al. Risk factors associated with preeclampsia in healthy nulliparous women. Am J Obstet Gynecol 1997;177:1003-10.

36 Yu CK, Smith GC, Papageorghiou AT, Cacho AM, Nicolaides KH, for the Fetal Medicine Foundation Second Trimester Screening Group. An integrated model for the prediction of preeclampsia using maternal factors and uterine artery Doppler velocimetry in unselected low-risk women. Am J Obstet Gynecol 2005;193:429-36.

37 Silva LM, Coolman M, Steegers EA, Jaddoe VW, Moll HA, Hofman A, et al. Low socioeconomic status is a risk factor for preeclampsia: the Generation R Study. J Hypertens 2008;26:1200-8.

38 Silva LM, Steegers EA, Burdorf A, Jaddoe VW, Arends LR, Hofman A, et al. No midpregnancy fall in diastolic blood pressure in women with a low educational level: the Generation R Study. Hypertension 2008;52:645-51.
39 Cnossen JS, Leeflang MM, de Haan EE, Mol BW, van der Post JA, Khan KS, et al. Accuracy of body mass index in predicting preeclampsia: bivariate meta-analysis. BJOG 2007;114:1477-85.

40 Skjaerven R, Vatten LJ, Wilcox AJ, Ronning T, Irgens LM, Lie RT. Recurrence of pre-eclampsia across generations: exploring fetal and maternal genetic components in a population based cohort. BMJ 2005;331:877.

41 Carr DB, Epplein M, Johnson CO, Easterling TR, Critchlow CW. A sister's risk: family history as a predictor of preeclampsia. Am J Obstet Gynecol 2005;193:965-72.

42 Rigo J Jr, Boze T, Derzsy Z, Derzbach L, Treszl A, Lazar L, et al. Family history of early-onset cardiovascular disorders is associated with a higher risk of severe preeclampsia. Eur J Obstet Gynecol Reprod Biol 2006;128:148-51.

43 Bellamy L, Casas JP, Hingorani AD, Williams DJ. Pre-eclampsia and risk of cardiovascular disease and cancer in later life: systematic review and meta-analysis BMJ 2007;335:974.

44 Dempsey JC, Williams MA, Luthy DA, Emanuel I, Shy K. Weight at birth and subsequent risk of preeclampsia as an adult. Am J Obstet Gynecol 2003;189:494-500.

45 Weiss JL, Malone FD, Vidaver J, Ball RH, Nyberg DA, Comstock CH, et al. Threatened abortion: a risk factor for poor pregnancy outcome, a population-based screening study. Am J Obstet Gynecol 2004;190:745-50.

46 Conde-Agudelo A, Althabe F, Belizan JM, Kafury-Goeta AC. Cigarette smoking during pregnancy and risk of preeclampsia: a systematic review. Am J Obstet Gynecol 1999;181:1026-35.

47 Klonoff-Cohen HS, Edelstein SL. Alcohol consumption during pregnancy and preeclampsia. J Women's Health 1996;5:225-30.

48 Barry D, Petry NM. Associations between body mass index and substance use disorders differ by gender: results from the National Epidemiologic Survey on Alcohol and Related Conditions. Addict Behav 2009;34:51-60.

49 Gearhardt AN, Corbin WR. Body mass index and alcohol consumption: family history of alcoholism as a moderator. Psychol Addict Behav 2009;23:216-25.

50 Poon LC, Kametas NA, Maiz N, Akolekar R, Nicolaides KH. Firsttrimester prediction of hypertensive disorders in pregnancy. Hypertension 2009;53:812-8.

51 Herraiz I, Arbues J, Camano I, Gomez-Montes E, Graneras A, Galindo A. Application of a first-trimester prediction model for preeclampsia based on uterine arteries and maternal history in high-risk pregnancies. Prenat Diagn 2009;29:1123-9.

52 National Institute for Health and Clinical Excellence. NICE clinical guideline 107. Hypertension in pregnancy. The management of hypertensive disorders during pregnancy. NICE,2010.

Accepted: 14 February 2011 